\title{
A rare cause of carpal tunnel syndrome in childhood: Benign recurrent intrahepatic cholestasis
}

\author{
Necati Balamtekin ${ }^{1 *}$, Nuray Uslu¹, Cagri Temucin², Hulya Demir¹, \\ Inci Nur Saltik Temizel ${ }^{1}$ \\ ${ }^{1}$ Hacettepe University, Faculty of Medicine, Department of Pediatrics, \\ Section of Gastroenterology, Hepatology and Nutrition, 06100 Ankara, Turkey \\ ${ }^{2}$ Hacettepe University, Faculty of Medicine, Department of Neurology, \\ 06100 Ankara, Turkey
}

Received 18 March 2009; Accepted 14 December 2009

\begin{abstract}
Carpal tunnel syndrome and benign recurrent intrahepatic cholestasis are rare conditions in childhood. Benign intrahepatic cholestasis is characterized by repeated self-limited attacks of cholestasis that can start at any age and last from weeks to months. The patients are asymptomatic between these attacks. We report a 16 year-old male patient with benign recurrent intrahepatic cholestasis who developed carpal tunnel syndrome during a cholestatic attack. He was admitted with complaints of jaundice, pruritus and pain, tingling and muscle weakness in both hands for 15 days. Nerve conduction studies revealed findings compatible with carpal tunnel syndrome. He was started on ursodeoxycholic acid, fat soluble vitamins and cholestyramine and cholestasis regressed after four weeks of therapy. With the improvement of cholestasis, the symptoms of carpal tunnel syndrome also disappeared. In conclusion, benign recurrent intrahepatic cholestasis can be a rare cause of carpal tunnel syndrome in childhood. We also advocate treating the underlying disease as an appropriate conservative treatment before surgery.
\end{abstract}

Keywords: Child • Carpal tunnel syndrome • Benign recurrent intrahepatic cholestasis

(C) Versita Sp. z 0.0 .

\section{Introduction}

Carpal tunnel syndrome (CTS) or median entrapment neuropathy is a rare condition during childhood. Etiologic factors for CTS include lysosomal storage diseases, connective tissue disorders, hereditary median neuropathy, neoplasms and direct injury. The lysosomal storage diseases are the most common causes of CTS in childhood. Open carpal tunnel release surgery is the main treatment for CTS [1].

Benign recurrent intrahepatic cholestasis (BRIC) is a rare cause of cholestasis in children. The disease is characterized by repeated self-limited attacks of cholestasis that can start at any age and last from weeks to months. The patients are asymptomatic between these attacks. Intense pruritus and jaundice are the hallmarks of the disease. Other common symptoms include malaise, irritability, nausea, vomiting, and anorexia. Less commonly, patients experience fever, arthralgias, headaches, and urticaria. Cirrhosis or liver failure due to BRIC is an unexpected condition. The defective gene (ATP8B1) causing BRIC has been mapped to the long arm of chromosome 18. The pathophysiology of the disease is poorly understood. Specific treatment is not available [2].

Herein, we report a male patient with BRIC who developed CTS following a cholestasis attack.

\section{Case Report}

A 16 year-old boy has been followed with the diagnosis of BRIC since the age of 21 months. He was admitted to our out-patient department with complaints of jaundice, pruritus for four weeks, and pain, tingling 
Table 1. The first and second nerve conduction studies (NCS) of right and left median nerves. Note the evident improvement in the compound muscle action potential (CMAP) and sensory nerve action potential (SNAP) of the right median nerve in the second NCS.

\begin{tabular}{|c|c|c|c|c|c|c|c|c|c|c|c|c|c|c|}
\hline & \multicolumn{7}{|c|}{ Right median nerve } & \multicolumn{7}{|c|}{ Left median nerve } \\
\hline & \multicolumn{3}{|c|}{ Motor NCS } & \multicolumn{4}{|c|}{ Sensory NCS } & \multicolumn{3}{|c|}{ Motor NCS } & \multicolumn{4}{|c|}{ Sensory NCS } \\
\hline & & & & \multicolumn{2}{|c|}{ Digit II-wrist } & \multicolumn{2}{|c|}{ Palm-wrist } & & & & \multicolumn{2}{|c|}{ Digit Il-wrist } & \multicolumn{2}{|c|}{ Palm-wrist } \\
\hline & & & CMAP & & SNAP & & SNAP & & & CMAP & & SNAP & & SNAP \\
\hline & MDL & NCV & Amp & NCV & Amp & NCV & Amp & MDL & NCV & Amp & NCV & Amp & NCV & Amp \\
\hline & (ms) & $(\mathrm{m} / \mathrm{s})$ & $(\mathrm{mV})$ & $(\mathrm{m} / \mathrm{s})$ & $(\mu \mathrm{V})$ & $(\mathrm{m} / \mathrm{s})$ & $(\mu \mathrm{V})$ & (ms) & $(\mathrm{m} / \mathrm{s})$ & $(\mathrm{mV})$ & $(\mathrm{m} / \mathrm{s})$ & $(\mu \mathrm{V})$ & $(\mathrm{m} / \mathrm{s})$ & $(\mu \mathrm{V})$ \\
\hline $1^{s t} N C S$ & 4,1 & 56 & 3,3 & - & Absent & - & Absent & 3 & 52 & 6,2 & 43 & 25 & 41 & 52 \\
\hline $2^{\text {nd }} N C S$ & 4 & 53 & 8,3 & 40 & 6,1 & 38 & 34 & 3,2 & 53 & 9,1 & 44 & 15 & 44 & 76 \\
\hline
\end{tabular}

NCS: Nerve conduction studies; MDL: Motor distal latency; NCV: Nerve conduction velocity; CMAP Compound muscle action potential; SNAP: Sensory nerve action potential

and muscle weakness in both hands for two weeks. It was his fourth cholestatic attack.

On physical examination, his body weight was $50 \mathrm{~kg}$ (25-50 percentiles) and height was $170 \mathrm{~cm}$ (75-90 percentiles). The child had generalized jaundice, itching scars, pain and muscle weakness in both hands. The liver was $1 \mathrm{~cm}$ palpable below the right costal margin. The rest of his physical examination was normal.

Laboratory findings revealed normal complete blood count. Total bilirubin was $39 \mathrm{mg} / \mathrm{dl}$ with a conjugated component of $38 \mathrm{mg} / \mathrm{dl}$. Alanine aminotransferase (ALT), aspartate aminotransferase (AST), gamma glutamyl transpeptidase (GGT) and alkaline phosphatase were $45 \mathrm{IU} / \mathrm{L}$ (normal <40), $54 \mathrm{IU} / \mathrm{L}$ (normal <35), $8.7 \mathrm{IU} / \mathrm{L}$ (normal 8-61) and $398 \mathrm{IU} / \mathrm{L}$ (normal 60-220), respectively. Serum total protein, albumin, ceruloplasmin and prothrombin time were within normal ranges. Lipid profile was normal. Viral serologies for hepatitis $A, B$, and $C$ were negative. Autoimmune markers including liver-kidney antimicrosomal antibodies (LKMA), anti-smooth muscle antibodies (ASMA), anti-nuclear antibodies (ANA) and double-strain DNA (ds-DNA) were negative. Minimal hepatomegaly and normal biliary tract were observed by abdominal ultrasonography. After informed consent was obtained, a liver biopsy was performed and the histological findings revealed centrilobular cholestasis. Due to his complaints in the hands, median and ulnar nerve conduction studies (NCS) (Key point equipment, Medtronic, Copenhagen) and electromyelography (EMG) were performed and the results were compatible with bilateral CTS. During the initial orthodromic studies, right median nerve sensory nerve action potentials (SNAP) from digit II-wrist and palm-wrist segments could not be obtained, but motor nerve compound muscle action potential (CMAP) was found to be low and distal motor latency (DML) was prolonged. Also, left median nerve sensory nerve conduction velocity (NCV) was mildly decreased, whereas motor NCS was normal. Ulnar nerve motor and sensory NCS were normal on both sides. In needle EMG examination, some fibrillation potentials were detected in the right abductor pollicis brevis muscle. These findings indicated CTS in both hands that was severe on the right side and mild on the left.

The patient was given ursodeoxycholic acid (UDCA, $20 \mathrm{mg} / \mathrm{kg} /$ day), fat soluble vitamins, hydroxyzin and cholestyramine (13.5 g/day). After four weeks of treatment, jaundice and pruritus of the patient regressed. With the improvement of cholestasis, CTS symptoms also disappeared after four weeks of treatment. Two and a half months after the first NCS, a second examination was performed. An apparent electrophysiological improvement was also observed, especially in the right median nerve NCS. At this time, right median nerve SNAP was obtained with slow NCV as well as median CMAP amplitude in the normal range, but its distal motor latency was still prolonged. Left median nerve sensory NCV was still mildly slow whereas motor CNAP amplitude was increased. Median nerve NCS are summarized in Table 1. The second NCS revealed normal left median motor NCS and normal ulnar nerve motor and sensory NCS on both sides. Due to recovery from CTS symptoms and NCS findings with the improvement of cholestasis, we considered BRIC as a cause of CTS in our patient.

\section{Discussion}

Benign recurrent intrahepatic cholestasis, a rare cause of cholestasis in children, is characterized by jaundice and pruritus. These signs may persist or recur throughout life but do not lead to chronic liver disease or cirrhosis $[2,3]$. The treatment of BRIC is purely symptomatic. There are conflicting reports regarding the effect of therapeutic alternatives such as UDCA, cholestyramin and rifampicin [3-5].

CTS is also rare in childhood. Although concomitance of Alagille syndrome and CTS has been described, BRIC has not been reported to be associated with 
CTS [6]. In our patient, cholestasis decreased and disappeared with UDCA and cholestyramin treatment within four weeks. Similarly, as cholestasis improved, the symptoms of CTS started to resolve in two weeks and disappeared in the fourth week of therapy.

In their patient with Alagille syndrome and CTS, Voermans et al. [6] suggested that years of rubbing both hands and wrists resulted in an increase in callosity and subcutaneous fibrotic changes in the palms of both hands, and intermittent external compression at the distal forearm eventually caused severe bilateral median neuropathy. This pathophysiologic mechanism is probably similar to that of occupational CTS, in which repetitive strain on the distal forearm or wrists intermittently causes a compression neuropathy [6].

Unlike the case of Voermans et al, the duration of cholestasis and pruritus was short in our case. However, in subacute CTS, the symptoms of nerve compression may appear for as short as one week [7]. Acute and

\section{References}

[1] Van Meir N, De Smet L. Carpal tunnel syndrome in children. J Pediatr Orthop B 2005; 14: 42-45

[2] Luketic VA, Shiffman ML. Benign recurrent intrahepatic cholestasis. Clin Liver Dis 2004; 8: 133-149

[3] Gupta V, Kumar M, Bhatia BD. Benign recurrent intrahepatic cholestasis. Indian J Pediatr 2005; 72 : 793-794

[4] Cançado EL, Leitao RM, Carrilho FJ, Laudanna AA. Unexpected clinical remission of cholestasis after rifampicin therapy in patients with normal or slightly increased levels of gamma-glutamyl transpeptidase. Am J Gastroenterol 1998; 93: 1510-1517

[5] Nicolas I, Pons JA, Vargas A, Gallardo F, Albaladejo A, Nicolas JM.. Ursodeoxycholic acid treatment shortens the course of cholestasis in two patients with benign recurrent intrahepatic cholestasis. Gastroenterol Hepatol 2003; 26: 421-423 chronic compression of a peripheral nerve can induce changes in the intraneuronal microcirculation and the nerve fiber structure, increase vascular permeability with subsequent edema formation, and impair anterograde and retrograde axonal transport, which all contribute to the clinical symptoms and deterioration of nerve function [8]. In our case, local trauma due to extreme itching and jaundice, and perhaps increased plasma bile acids, might have disturbed neuronal microcirculation and function. Although the exact mechanism of CTS in BRIC is not known, the subsiding of CTS symptoms with regression of cholestasis supports our hypothesis.

In conclusion, a patient with cholestasis or BRIC who has symptoms such as pain, tingling and muscle weakness in the hands may have CTS. Moreover, since treatment of cholestasis may also improve the CTS, we imply that in such cases, it could be an adequate conservative treatment before surgery.

[6] Voermans NC, van Alfen N, Tolboom JJ, Koetsveld AC, Sie LT. Pediatric median neuropathy due to pruritus in Alagille syndrome. Pediatric Neurology 2006; 35: 216-219

[7] Figus A, Iwuagwu FC, Elliot D. Subacute nerve compressions after trauma and surgery on the hand. Plast Reconstr Surg 2007; 120: 705-712

[8] Dahlin LB. Aspects on pathophysiology of nerve entrapments and nerve compression injuries. Neurosurg Clin N Am 1991; 2: 21-29 